\title{
c-Fos/ERK promotes the progression from pancreatic intraepithelial neoplasia to pancreatic ductal adenocarcinoma
}

\author{
LEI YOU* ${ }^{*}$ XIAOXIA REN* ${ }^{*}$ YONGXING DU, WENJING ZHAO, \\ MING CUI, GE CHEN and YUPEI ZHAO
}

\begin{abstract}
Department of General Surgery, Peking Union Medical College Hospital, Chinese Academy of Medical Sciences and Peking Union Medical College, Beijing 100730, P.R. China
\end{abstract}

Received May 19, 2016; Accepted October 3, 2016

DOI: $10.3892 /$ or.2016.5169

\begin{abstract}
Pathogenesis of pancreatic ductal adenocarcinoma (PDAC) is thought to develop through the progression of precursor lesions, known as pancreatic intraepithelial neoplasias (PanIN). In the present study, we showed that c-Fos promoted proliferation, cell cycle and migration in pancreatic cancer cells. Caerulein was used to accelerate the pathogenesis of Pdx-cre; Kras ${ }^{\mathrm{G} 12 \mathrm{D}}$ mice. During PanIN formation and development of PDAC, the expression of ERK and c-Fos increased concomitantly. When ERK activity was inhibited by U0126, the expression of c-Fos also decreased. Inactivation of ERK/c-Fos suppressed pancreatic lesions concurrently through proliferation, inflammation and apoptosis. Our findings suggest that the ERK/c-Fos pathway is required for PDAC initiation and progression.
\end{abstract}

\section{Introduction}

Pancreatic ductal adenocarcinoma (PDAC) is the fourth leading cause of cancer deaths worldwide with a 5-year survival rate of less than $7 \%$, a mortality rate that is nearly identical to the incidence rate $(1,2)$. The main reason for the poor survival rate is that the current diagnostic methods lack the sensitivity and the specificity to identify markers of PDAC in the early stages, resulting in delayed diagnosis until the later stages of the disease. In 2015, fewer than $20 \%$

Correspondence to: Professor Ge Chen or Professor Yupei Zhao, Department of General Surgery, Peking Union Medical College Hospital, Chinese Academy of Medical Sciences and Peking Union Medical College, No. 1 Shuai Fu Yuan, Dongcheng, Beijing 100730, P.R. China

E-mail: chenge@pumch.cn

E-mail: zhao8028@263.net

\section{*Contributed equally}

Key words: C-Fos, pancreatic intraepithelial neoplasia, pancreatic ductal adenocarcinoma, Pdx-cre; Kras $^{\mathrm{G} 12 \mathrm{D}}$ mice of newly diagnosed patients with pancreatic cancer were classified as having resectable cancer (3). Therefore, it is necessary to characterize PDAC's precursor lesions and the pathological mechanisms regulating the genetic progression from normal cells to PDAC (4).

Pancreatic intraepithelial neoplasia (PanIN) is the most common pancreatic precursor lesion. Based on increasing degrees of architectural and nuclear atypia, PanIN are grouped into three histological stages: PanIN-1 (PanIN-1A and PanIN-1B), PanIN-2 and PanIN-3 (5). Activating mutations in the KRAS oncogene are found in all three PanIN stages and over $90 \%$ of invasive PDAC (6). A genetically engineered mouse model, Pdx-cre; Kras ${ }^{\mathrm{G} 12 \mathrm{D}}$, which uses a $\mathrm{Pdx} 1$ promoter to drive the expression of mutant Kras, is able to recapitulate the progression of human PanIN to PDAC successfully and serves as a convenient tool to explore the precursor lesions and the pathological mechanisms of the pancreatic cancer (7).

AP-1 component members, c-Fos and c-Jun, participate in the regulation of many processes, including proliferation, cell cycle progression, migration, differentiation, apoptosis and angiogenesis $(8,9)$. In addition to the specific role of c-Fos as a differentiation transcription factor for osteoclasts and bone resorbing cells, c-Fos also has an important role in the tumor formation and suppression. It has been reported that c-Fos downregulation in MCF-7/ADR cells results in enhanced apoptosis and altered expression of apoptosis-associated proteins, including Bax, Bcl-2, p53 and PUMA (10). The ERK/c-Fos/MMP-7 pathway plays an important role in modulating the invasion of colon cancer cells and inhibition of this pathway holds promise as a treatment for the colon cancer metastasis (11).

In the present study, we investigated the role of AP-1 in initiating the PanIN-PDAC progression in vivo using Pdx-cre; Kras $^{\mathrm{G} 12 \mathrm{D}}$ mouse model. Our results indicate that the c-Fos expression, but not the c-Jun expression, increased during the development from precursor lesions to tumor. We demonstrated that ERK activates c-Fos to promote the PanIN-PDAC progression through initiation of proliferation, inflammation and apoptosis. These results will help to characterize the early prognosis of the pancreatic cancer. 


\section{Materials and methods}

Cell culture and transfection. Cell lines were obtained from the Cell Resource Center in Peking Union Medical College (PUMC; Beijing, China). Panc-1 cells were cultured in Dulbecco's modified Eagle's medium (DMEM; HyClone Laboratories; Thermo Fisher Scientific) with 10\% fetal bovine serum (FBS) and PCT-3 cells were cultured in RPMI-1640 (HyClone Laboratories; Thermo Fisher Scientific) with 10\% FBS in $5 \% \mathrm{CO}_{2}$ at $37^{\circ} \mathrm{C}$. Adherent cells were passaged every 2-3 days with $0.5 \mathrm{mg} / \mathrm{ml}$ trypsin (1:250) and $0.53 \mathrm{mM}$ ethylenediaminetetraacetic acid (EDTA). siRNA oligonucleotides were designed against c-Fos as follows: GGGGCAAGGTGGA ACAGTTAT. Cells were transfected using Lipofectamine ${ }^{\mathrm{TM}}$ 2000 (Invitrogen, Carlsbad, CA, USA).

Western blot analysis. Proteins were extracted with SDS lysis buffer [50 mM Tris-HCl (pH 6.8), 10\% glycerol and 2\% SDS] and quantified using the BCA protein assay reagent (Thermo Fisher Scientific).Extracts were separated on a $12 \%$ SDS-PAGE gel and electrophoretically transferred to PVDF membrane (GE Healthcare). The membrane was blocked in 5\% skim milk for $1 \mathrm{~h}$ at room temperature and then incubated overnight with the indicated antibodies at $4^{\circ} \mathrm{C}$. Antibodies against c-Fos $(1: 1,000)$ and GAPDH $(1: 3,000)$ were purchased from Cell Signaling Technology (Danvers, MA, USA). The membrane was incubated with an anti-rabbit or an anti-mouse IgG-HRP (Santa Cruz Biotechnology, Santa Cruz, CA, USA) for $1 \mathrm{~h}$ at room temperature. Chemiluminescence was detected using an ECL blot detection system (Santa Cruz Biotechnology).

Cell proliferation assay. Cell proliferation was assessed by the CCK-8 assay (Dojindo Molecular Technologies). Cells were seeded at a density of 2,000 cells/well in 96-well plates. A total of $10 \mu$ CCK-8 solution was added to each well containing $100 \mu \mathrm{l}$ of culture medium and incubated for $2 \mathrm{~h}$ at $37^{\circ} \mathrm{C}$. Absorbance was measured at $450 \mathrm{~nm}$ using a multiwell spectrophotometer (BioTek Instruments, Inc., Winooski, VT, USA).

Cell cycle assay. Cells were transfected in 6-well plates $\left(5 \times 10^{5}\right.$ cells/well). After $48 \mathrm{~h}$, cells were harvested, washed with cold phosphate-buffered saline (PBS) and fixed in $70 \%$ ethanol overnight at $4^{\circ} \mathrm{C}$. After centrifugation at $800-1,000 \mathrm{rpm}$ three times, cells were re-suspended with $500 \mu 1$ PBS. Cells were stained with a solution containing $10 \mathrm{mg} / \mathrm{ml}$ RNase A, $0.1 \%$ Triton X-100 and $1 \mathrm{mg} / \mathrm{ml}$ propidium iodide. Cell cycle analysis was performed by fluorescence-activated cell sorting.

Wound-healing assay. Cells were transfected in 6-well plates $\left(5 \times 10^{5}\right.$ cells/well). After $24 \mathrm{~h}$, a monolayer of cells was scratched by a $200 \mathrm{ml}$ tip in serum-free medium. Cell migration was quantified by the area of migrated cells to the scratched cell-free zone after $24 \mathrm{~h}$, and measured by ImageJ software.

Mouse model and in vivo treatment. The genetically engineered mouse stains Pdx1-cre and LSL-Kras ${ }^{\mathrm{G} 12 \mathrm{D}}$ used in the present study were purchased from the National Cancer Institute (NCI; Rockville, MD, USA). Pdx-cre; $\mathrm{Kras}^{\mathrm{G} 12 \mathrm{D}}$ mice were generated by crossing LSL-Kras ${ }^{\mathrm{G} 12 \mathrm{D}}$ mice with Pdx1-cre mice. The mutant mice were genotyped by PCR using primers as followed: lox sense primer: AGCTAGCCACCATGGCTT GAGTAAGTCTGCA and $l o x$ antisense primer: CCTTTACA AGCGCACGCAGACTGTAGA; cre sense primer: CTGGA CTACATCTTGAGTTGC and cre antisense primer: GGTG TACGGTCAGTAAATTTG. Animals were housed in a clean vivarium and fed standard mouse chow. To accelerate carcinogenesis in Pdx-cre; $\mathrm{Kras}^{\mathrm{G} 12 \mathrm{D}}$ mice, an intraperitoneal injection of caerulein $(50 \mu \mathrm{g} / \mathrm{kg} / \mathrm{day})$ was administered. ERK/c-Fos signaling blockade was accomplished using the inhibitor U0126 $(500 \mu \mathrm{g} / \mathrm{kg})$ every two days.

Animals were housed in the Experimental Animal Center of Peking Union Medical College Hospital. They were maintained in a 12-h light/dark cycle and temperature-controlled environment with ad libitum access to water and food. Animals were cared for and studies were performed in accordance with the principles of the 3Rs (replacement, reduction and refinement) and guidelines of the National and Beijing Experimental Animal Welfare Ethics.

Immunohistochemical analyses. Freshly isolated biopsies of the pancreas were fixed in $10 \%$ neutral-buffered formulin (Sigma-Aldrich) for $16 \mathrm{~h}$ and embedded in paraffin. Next, $5-\mu \mathrm{m}$-thick sections were deparaffinized, rehydrated and treated in boiling sodium citrate buffer $(10 \mathrm{mM}$ pH 6.0) for $10 \mathrm{~min}$ to unmask antigens. Endogenous peroxidase activity was quenched by treating the slides with 3\% hydrogen peroxide for $10 \mathrm{~min}$. Sections were blocked in PBS containing 10\% goat serum, $1 \%$ BSA and $0.1 \%$ Triton $\mathrm{X}-100$ for $1 \mathrm{~h}$ at room temperature prior to being incubated overnight at $4^{\circ} \mathrm{C}$ with antibodies: JNK (ab179461, Abcam, 1:100), p38 (ab170099, Abcam, 1:50), caspase-3 (ab13847, Abcam, 1:500), CK19 (10712-1-AP, Proteintech, 1:100), MMP9 (ab38898, Abcam, 1:100), ERK1/2 (\#4695, Cell Signaling Technology, 1:100), amylase (12540-1-AP, Proteintech, 1:100), CD45 (ab10558, Abcam, 1:100). After incubation with secondary biotinylated antibodies (1:200; KPL), the presence of the antigens was revealed using diaminobenzadine tetrachloride (DAB; Dako) and counter stained with nuclear red or with heamatoxylin (blue). The relative quantities of IHC reaction were accessed by Image-Pro Plus 6.0 .

Statistical analyses. Statistical analyzes were performed using the Student's t-test (two-tailed) in Microsoft Excel software (Microsoft, Redmond, WA, USA). The results are presented as the mean \pm standard deviation of triplicates of each experiment. All experiments were performed in triplicate unless stated otherwise. $\mathrm{P}<0.05$ were considered statistically significant.

\section{Results}

C-Fos promotes proliferation, cell cycle and migration in human pancreatic cancer cells. To examine the effects of c-Fos on pancreatic cancer cell growth, we first suppressed endogenous c-Fos expression in Panc-1 and PCT-3 cells by transfecting cells with siRNA against c-Fos (Fig. 1A). The results of the cell proliferation assay showed that c-Fos knockdown significantly inhibited cell growth (Fig. 1B). Flow 
A

Panc-1

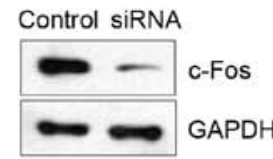

CT-3

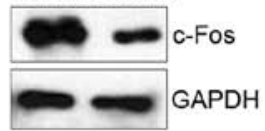

Control siRNA
B

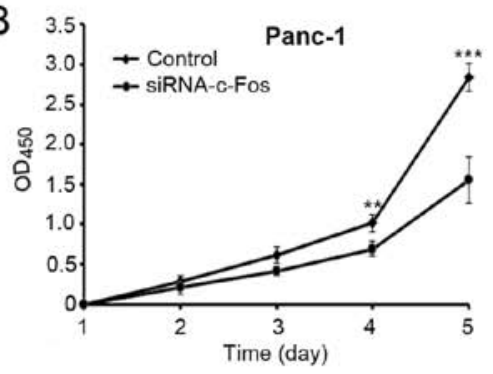

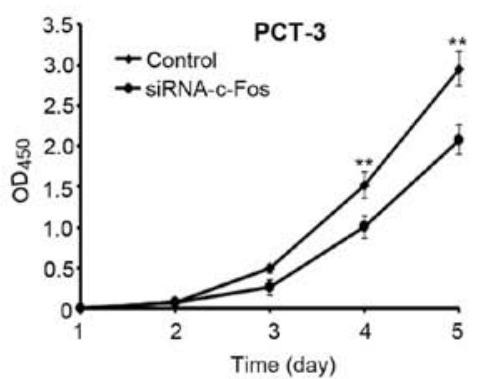

C
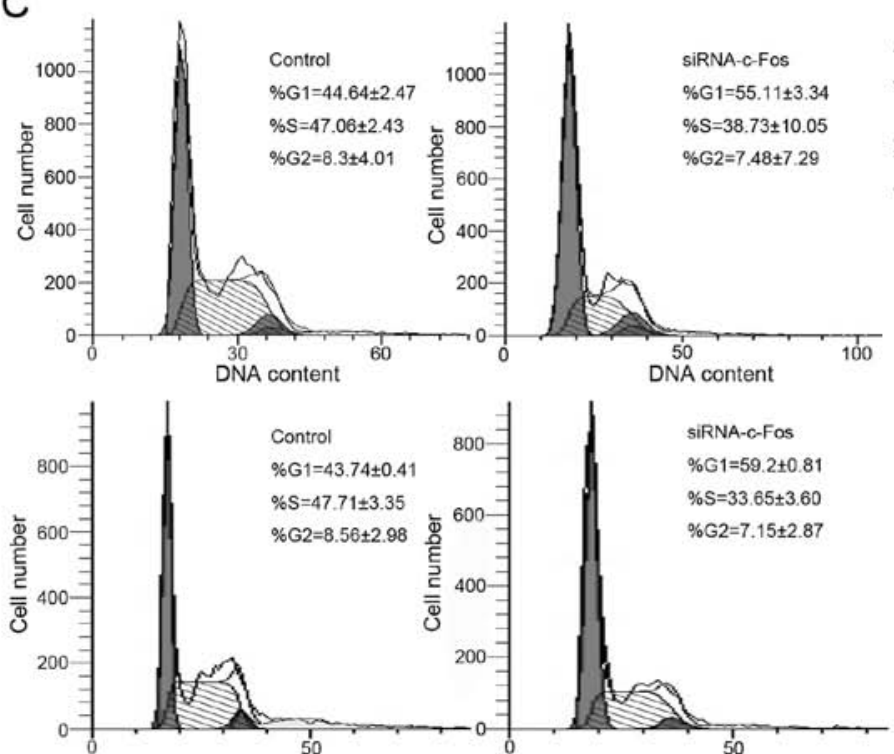

DNA content

D

$\mathrm{Oh}$

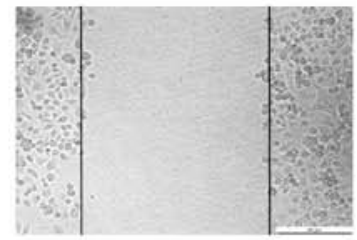

$24 \mathrm{~h}$

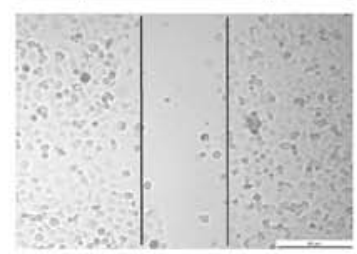

Control

$\mathrm{Oh}$

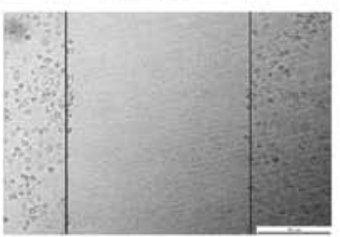

$24 \mathrm{~h}$

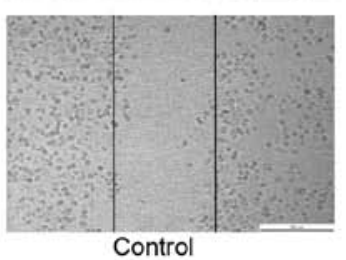

DNA content

SIRNA-C-Fos
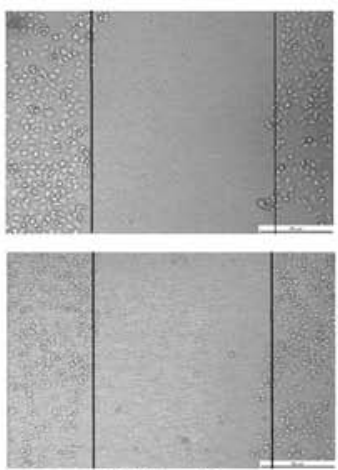

siRNA-C-Fos

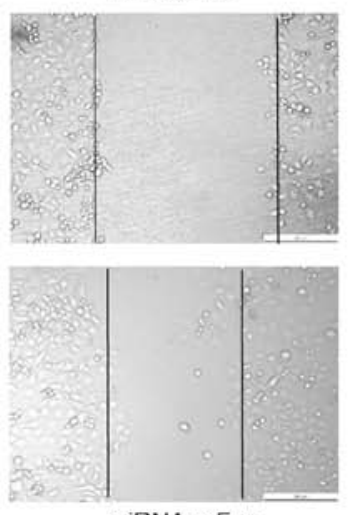

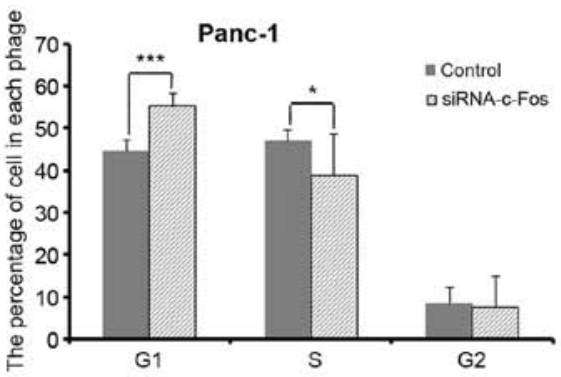

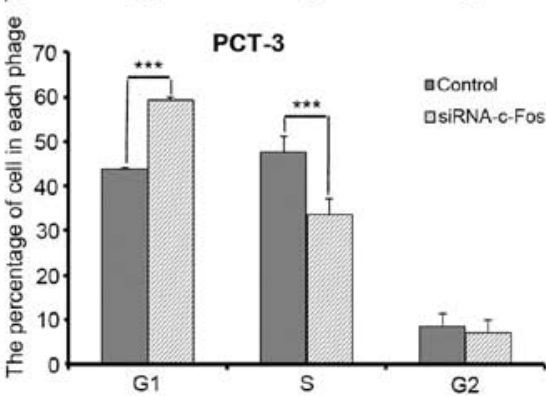

Panc-1

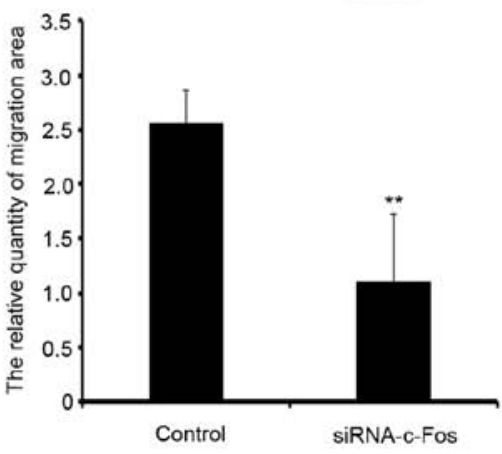

PCT-3

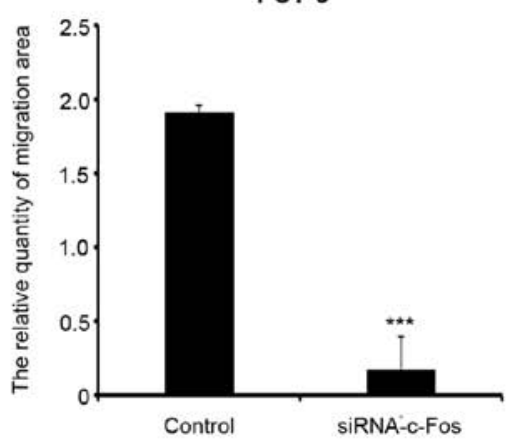

Figure 1. The effect of c-Fos on cell phenotype in human pancreatic cancer cells. (A) Knockdown of c-Fos. c-Fos siRNA or non-targeting siRNA were transfected into Panc-1 and PCT-3 cells for $48 \mathrm{~h}$, and cells were extracted for western blot analysis. (B) Cell proliferation assay. c-Fos siRNA or non-targeting siRNA were transfected into Panc-1 and PCT-3 cells for $24 \mathrm{~h}$, and cell survival was determined by CCK-8 assay. (C) Cell cycle assay. After transfection with c-Fos siRNA or non-targeting siRNA for $48 \mathrm{~h}$, the cells were harvested for cell cycle assay using flow cytometry. (D) Wound-healing assay. After transfection with c-Fos siRNA or non-targeting siRNA for $24 \mathrm{~h}$, cells were scratched with a $200 \mathrm{ml}$ tip to remove the monolayer. Cell migration was quantified by the area of migrated cells to the scratched cell-free zone after $24 \mathrm{~h}$. All experiments were run in triplicate, and the error bars represent the standard deviations of the triplicate samples. 

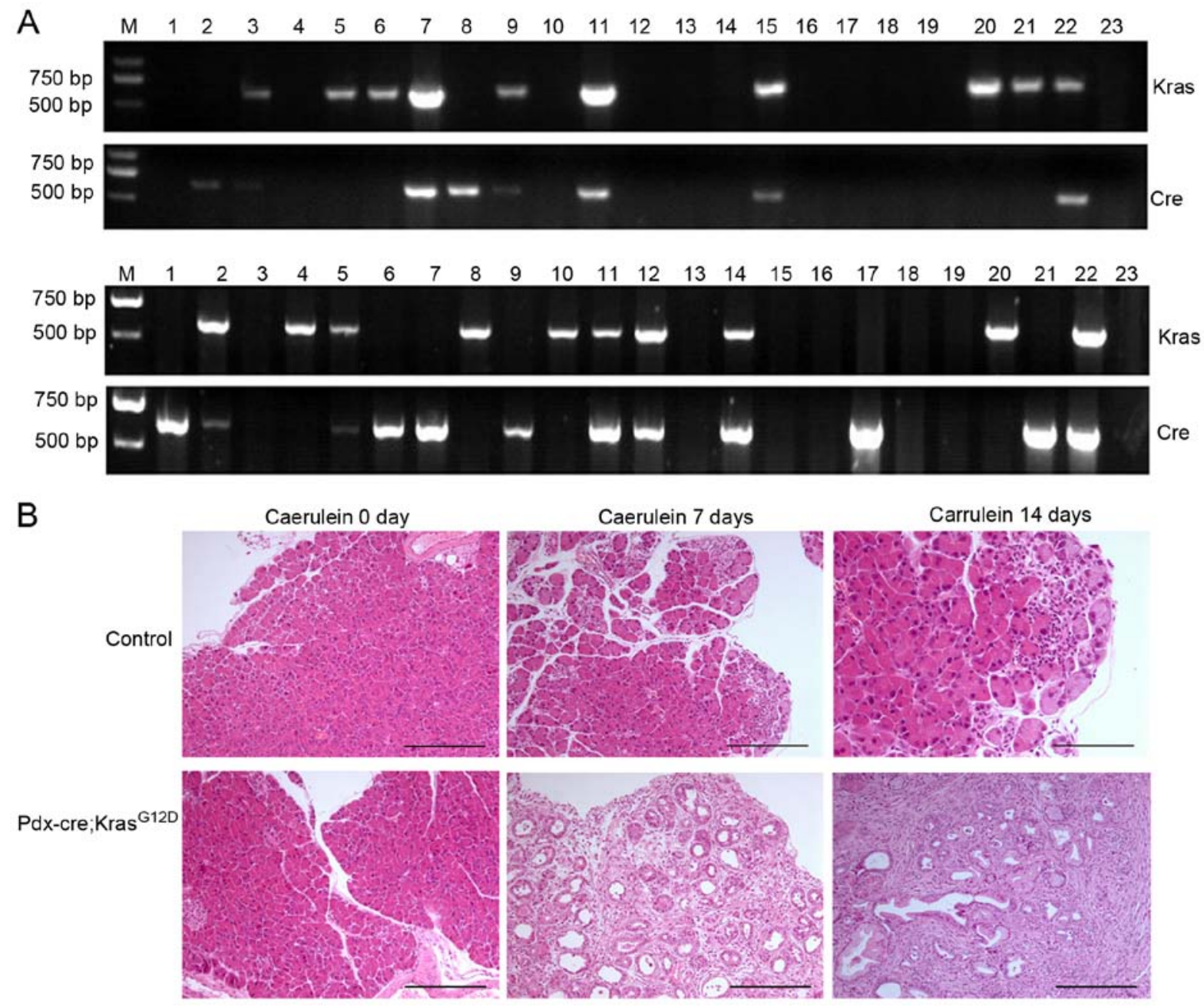

Figure 2. Characterization of the PK mouse model. (A) PCR of DNA from PK mice. DNA was isolated from mice generated by crossing LSL-Kras ${ }^{\mathrm{G} 12 \mathrm{D}}$ mice with Pdx1-cre mice and subjected to PCR. (B) Histological characteristics of pancreas from control or PK mice treated by caerulein for 7 days or 14 days were examined by H\&E (magnification, $\mathrm{x} 100)$.

cytometric analysis was used to test cell cycle progression. Our results show that the percentage of G1 phase cells was increased following knockdown of c-Fos expression (Fig. 1C). Wound-healing assay was performed to examine the effects of c-Fos on migration. Reduction in c-Fos levels decreased cell migration compared with the control group (Fig. 1D).

Characterization of the mouse model. $\mathrm{Pdx}-\mathrm{cre} ; \mathrm{Kras}^{\mathrm{G} 12 \mathrm{D}}$ (PK) mice are attained by crossing LSL-Kras ${ }^{\mathrm{G} 12 \mathrm{D}}$ mice with Pdx-cre mice. To identify transgenic mice, DNA was isolated and PCR amplification of both lox and cre genes indicated that the recombinant mouse model was established successfully (Fig. 2A). The positive rate for recombinant mice was $<20 \%$. The pancreas from PK mice is larger than their littermate controls and have more nodules, particularly those treated by caerulein for a longer period of time. Immunohistochemistry (IHC) revealed that the pancreas of PK mice treated by caerulein for 7 days developed PanIN-1 to PanIN-2 lesions, and those treated by caerulein for 14 days developed a higher degree of PanIN lesions relative to PDAC (Fig. 2B). In contrast, the pancreas from control mice developed few ductal lesions with the exception of some inflammatory infiltration after caerulein treatment for 7 or 14 days.
The expression of ERK/c-Fos increased during PDAC initiation and progression. C-Jun and c-Fos are components of the AP-1 complex, which is involved in numerous cell activities including proliferation, apoptosis, survival, tumorigenesis and tissue morphogenesis (12). To characterize the function of AP-1 in PDAC tumorigenesis, we examined the expression of both c-Jun and c-Fos in the pancreas of PK mice. Compared with the control mice, the expression level of c-Fos, but not c-Jun, was enhanced in PanIN lesions of caerulein-treated PK mice and progressively increased as pancreatic lesion stage progressed (Fig. 3A). AP-1 is reportedly involved in the downstream regulation of mitogen activated protein kinase (MAPK) $(13,14)$; therefore, we examined the expression of ERK, JNK and p38 in PK mice. As shown in Fig. 3B, the expression of ERK is similar to c-Fos levels, whereas JNK and p38 showed little difference in expression. Therefore, the expression of ERK/c-Fos increased during PDAC initiation and progression.

ERK/C-Fos is required for PDAC initiation and progression. As ERK/c-Fos is highly expressed in pancreatic cancer, we postulated that ERK/c-Fos is required for tumorigenesis. To test this hypothesis, we treated 6-week-old PK mice with the ERK inhibitor, U0126, with or without caerulein for 
A

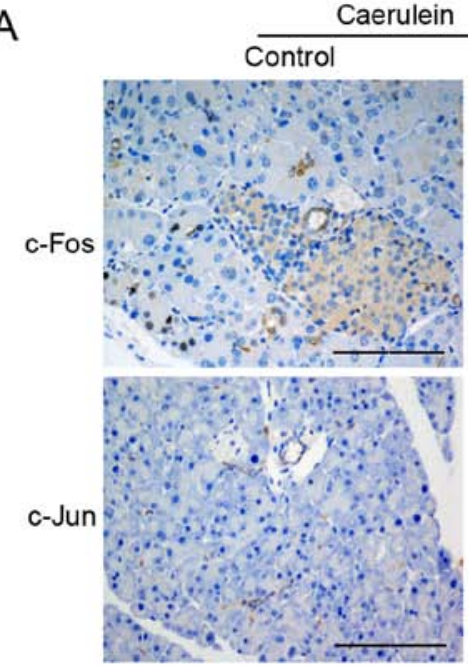

days 2 Pdx-cre;Kras ${ }^{\mathrm{G} 2 \mathrm{D}}$

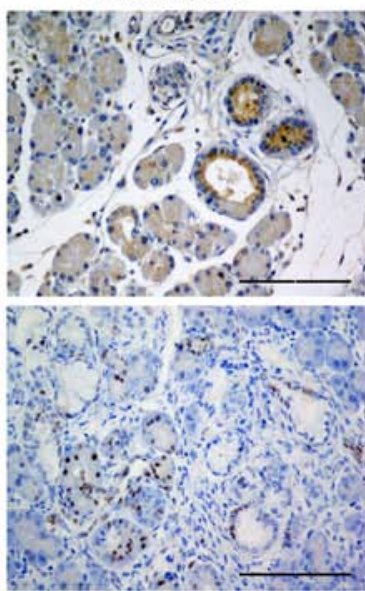

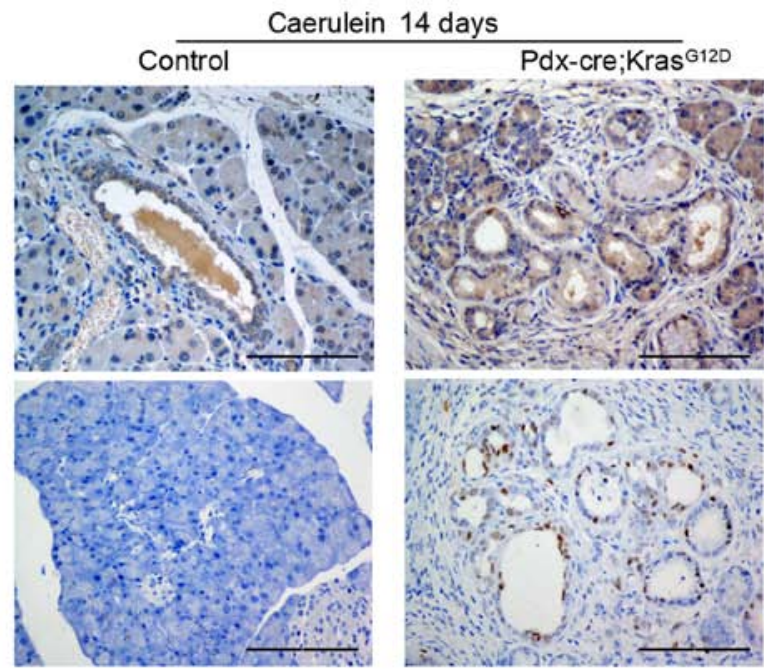

Caerulein 14 days

B

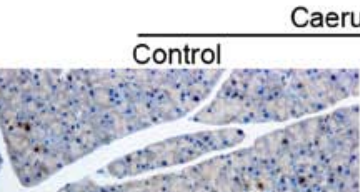

ERK1/2
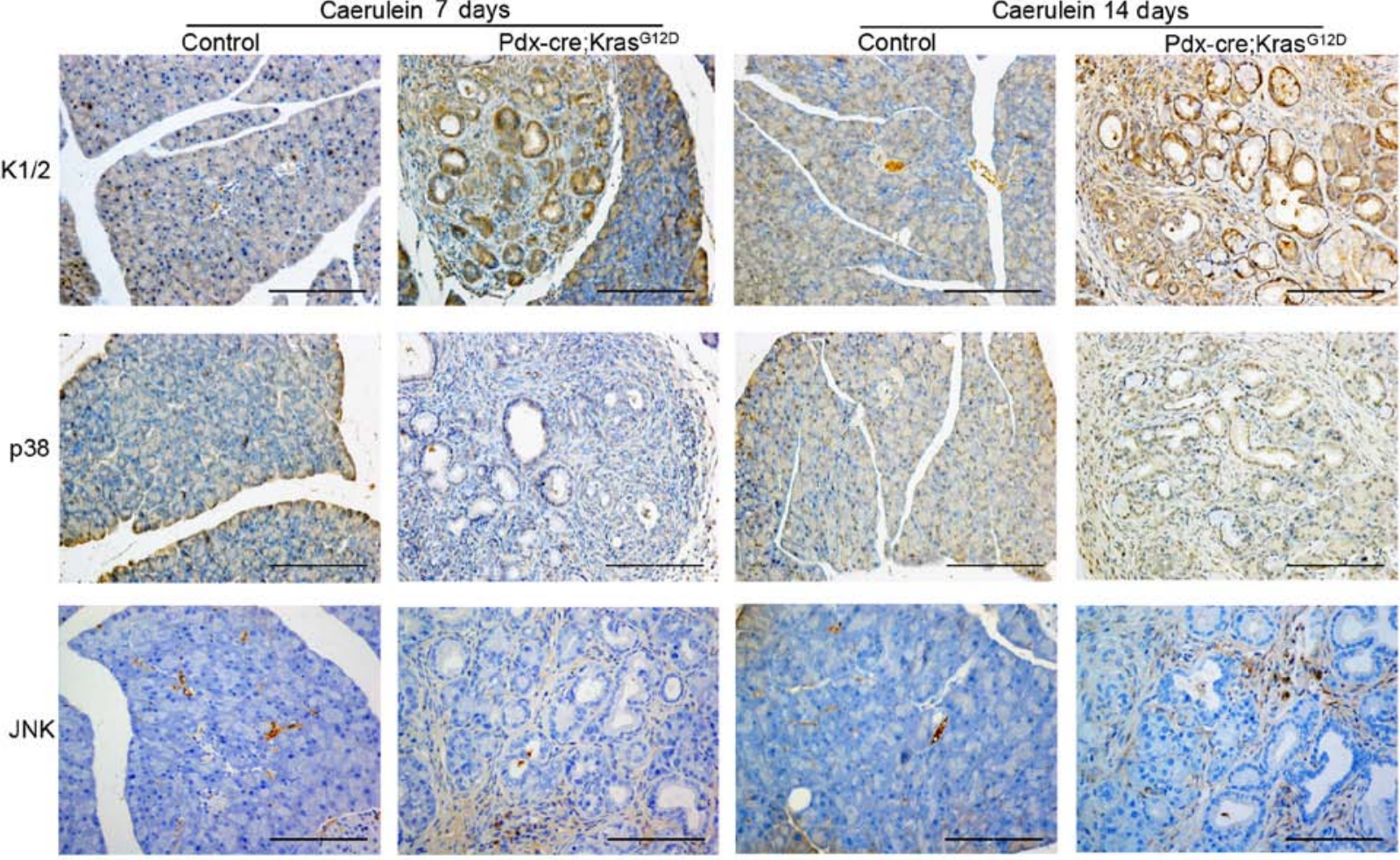

Figure 3. MAPK and AP-1 pathways in PK mice. The expression of c-Fos and c-Jun (A), ERK, JNK and p38 (B) in the pancreas from control or PK mice treated by caerulein for 7 days or 14 days was determined using IHC (magnification, $\mathrm{x} 200$ ).

15 days. U0126 reduced the expression of ERK and c-Fos and suppressed pancreatic ductal lesions (Fig. 4A). Furthermore, after 15 days of U0126+caerulein injection, the exocrine compartment of PK mice was partially replaced by fibrosis and ductal structures, as evident by the expression level of acinar marker amylase and duct marker cytokeratin 19 (CK19) (Fig. 4B). In contrast, the pancreas of PK mice treated with only caerulein displayed markedly more CK 19-positive duct structures and fewer amylase-positive parenchyma.

Suppression of ERK/c-Fos attenuates inflammation and proliferation, but promotes apoptosis. To determine the role of ERK/c-Fos in PanIN formation and development of PDAC, we tested additional markers of tumor phenotype. Levels of proliferation marker Ki-67 and inflammatory marker CD45 expression were substantially higher in PanIN and PDAC from PK mice compared to control mice, whereas the apoptosis marker, caspase-3, exhibited the opposite results (Fig. 5). Immunohistochemistry data also suggested that U0126 inhibits Ki-67 and CD45 expression and increased the levels of caspase-3. Therefore, the suppression of ERK/c-Fos attenuates inflammation and proliferation but promotes apoptosis to repress PanIN and PDAC progression.

\section{Discussion}

PDAC remains a highly lethal disease $(15,16)$. The posterior location of pancreas, which is in close proximity to duodenum, 


\section{A}
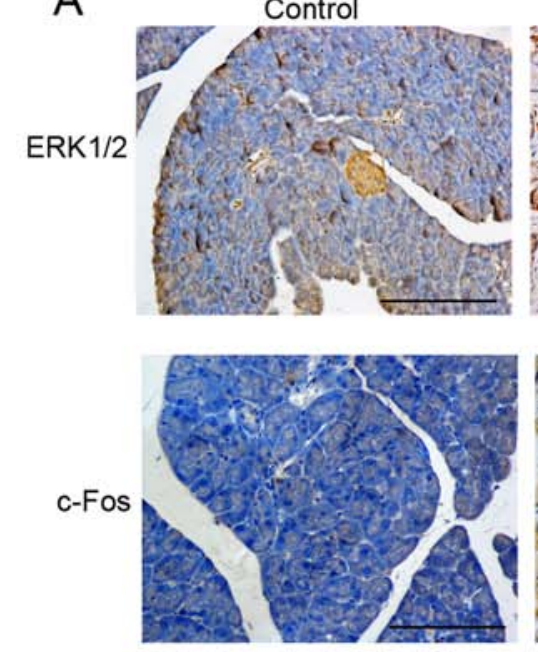

B
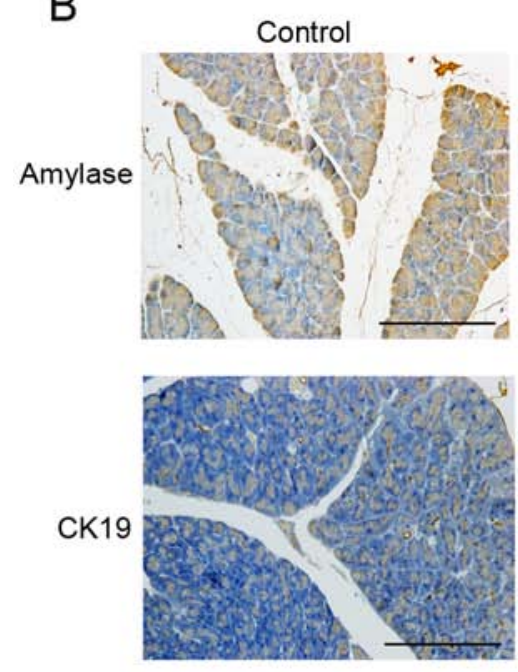
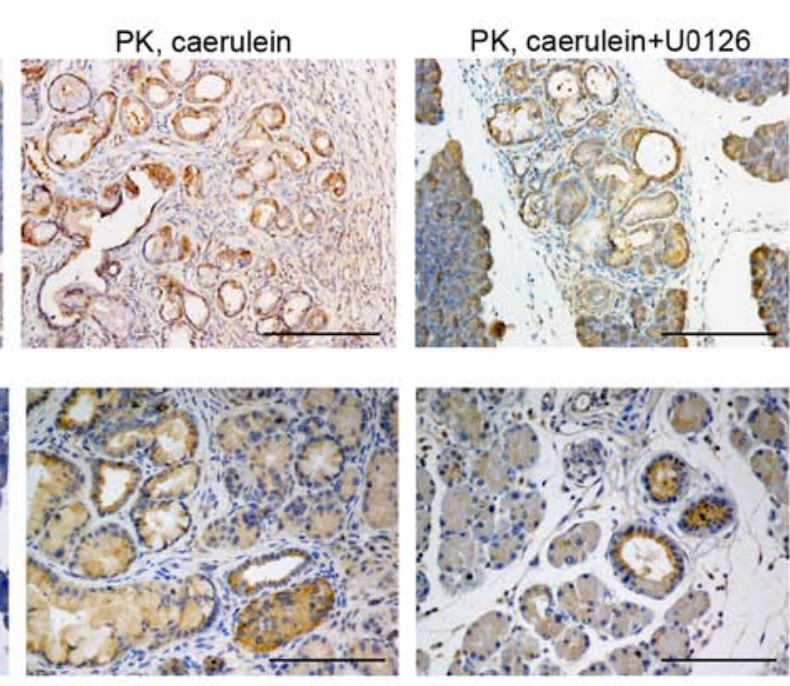

PK, caerulein
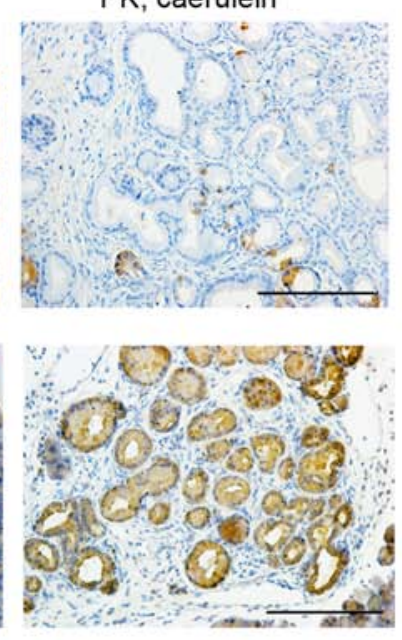
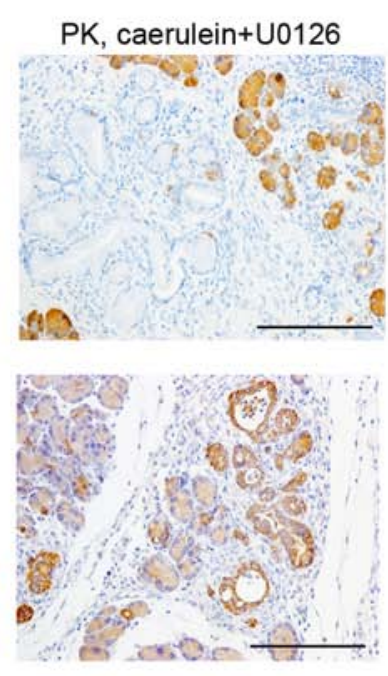

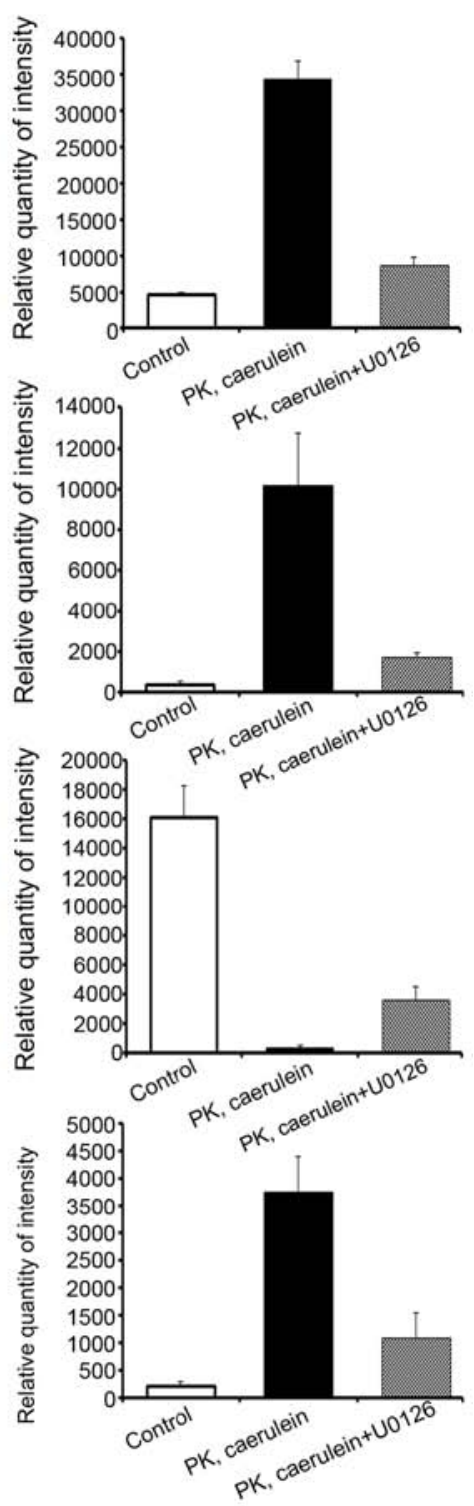

Figure 4. The effect of U0126 on PanIN-PDAC development. After 15 days of treatment with U0126+caerulein or caerulein only, the pancreas from mice were subjected to IHC (magnification, x200) to test the expression of c-Fos and c-Jun (A), amylase and CK19 (B).

common bile duct, celiac plexus, superior mesenteric artery (SMA), and portal vein, contributes to late diagnosis, as well as the bothersome symptoms of obstruction of biliary drainage, including infection, pain, chemotherapy resistance and unresectable pancreatic cancer (17-20). A genetically engineered mouse model is an appropriate tool to investigate the pathological mechanisms and early diagnosis of pancreatic cancer.

The Pdx-cre; Kras ${ }^{\mathrm{G} 12 \mathrm{D}}$ (PK) mouse model, which targets pancreas-specific expression of mutated Kras, recapitulates the human PanIN-to-PDAC sequence (7). PanIN lesions begin to appear at approximately 2 months, and high-grade PanINs are observed at 5 months. The progression from PanIN to invasive and metastatic PDAC occurs over several months, which is not conducive for a research study. Chronic pancreatitis has been identified as risk factor for PDAC development in humans and has been shown to significantly accelerate PanIN and PDAC development in Kras-driven mouse models $(21,22)$. Acute pancreatitis can progress to chronic pancreatitis in human patients under certain conditions (23). Several studies have also shown that acute pancreatitis markedly accelerates PanIN and PDAC development in Kras-driven mouse models (24). In response to acute pancreatitis induced by the cholecystokinin analog caerulein, acini transiently dedifferentiated into ductlike structures. Mutant Kras compromises the ability of acinar cells to regenerate following acute pancreatitis and locks damaged cells in a persistently dedifferentiated ductal state that can rapidly give rise to PanINs $(25,26)$. Thus, caeruleininduced pancreatitis provides a permissive environment for Kras-driven neoplasia. In the present study, the pancreas of PK mice treated by caerulein for 7 days developed PanIN-1 to PanIN-2 lesions, and PK mice treated by caerulein for 14 days developed a higher degree of PanIN lesions relative to PDAC. This improved PK mouse model allowed us to study PanIN to PDAC development on an accelerated timeline.

MAPKs are a family of serine-threonine protein kinases involved in many cellular processes including cell proliferation, 

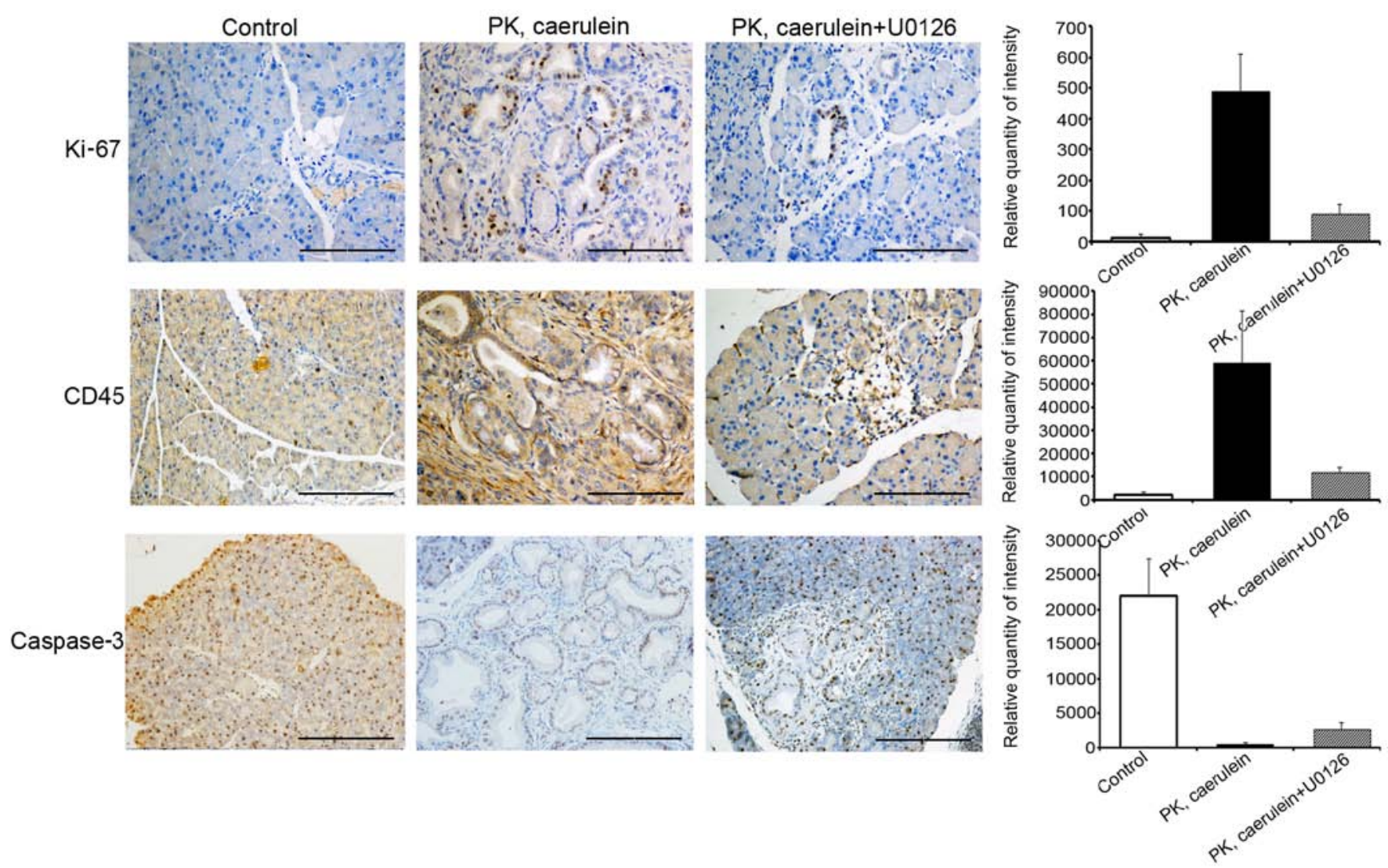

Figure 5. The effect of U0126 on proliferation, inflammation and apoptosis in PK mice. Mice were injected with U0126+caerulein or caerulein only, for 15 days and IHC (magnification, x200) was used to examine the level of Ki-67, CD45 and caspase-3.

differentiation, inflammation and cell death (27). Activation of several MAPKs, including ERK, p38 and c-Jun N-terminal kinase (JNK) are able to stimulate AP-1 (28). The results in PK mice suggested that the expression of ERK and c-Fos increased during PanIN formation and progression to PDAC, whereas the level of $\mathrm{p} 38$, JNK and c-Jun remained unchanged. In addition to the Kras gene, inactivation of other genes are involved in the pathophysiology of PDAC, including INK4A, TP53, SMAD4 or BRCA2 (29). Additionally, several signaling pathways may positively or negatively modulate the PanIN and PDAC development, including TGF $\alpha$, Hedgehog, Notch, EGFR and STAT (30-32). Here we provided evidence that the ERK/c-Fos pathway is essential for Kras-driven PDAC. Furthermore, our experiment showed that the ERK/c-Fos inhibitor U0126 suppressed acinar-ductal metaplasia, proliferation and inflammation and promoted apoptosis, leading to inhibition of pancreatic ductal lesions.

In summary, our results demonstrate that c-Fos induced cell growth, cell cycle and migration in the the pancreatic cancer cells. The in vivo experiments revealed that the expression of c-Fos, and the upstream transcription factor ERK, increased during PanIN formation. Additionally, the ERK/c-Fos inhibitor, U0126, suppressed the PanIN/PDAC progression initiation through proliferation, inflammation and apoptosis. Our findings suggest that the ERK/c-Fos pathway is required for PanIN formation and progression to PDAC, which will help to characterize the early prognosis of pancreatic cancer.

\section{Acknowledgements}

The present study was supported by grant for the Research Special Fund for Public Welfare Industry of Health (201402001), the Research Fund for the Doctoral Program of Higher Education (20131106110008) and the Research Fund for the Doctoral Program of Higher Education (20121106120048).

\section{References}

1. Siegel RL, Miller KD and Jemal A: Cancer statistics, 2016. CA Cancer J Clin 66: 7-30, 2016.

2. Ligat L, Saint-Laurent N, El-Mrani A, Gigoux V, Al Saati T, Tomasini R, Nigri J, Dejean S, Pont F, Baer R, et al: Pancreatic preneoplastic lesions plasma signatures and biomarkers based on proteome profiling of mouse models. Br J Cancer 113: 1590-1598, 2015.

3. Heestand GM, Murphy JD and Lowy AM: Approach to patients with pancreatic cancer without detectable metastases. J Clin Oncol 33: 1770-1778, 2015.

4. Shen R, Wang Q, Cheng S, Liu T, Jiang H, Zhu J, Wu Y and Wang L: The biological features of PanIN initiated from oncogenic Kras mutation in genetically engineered mouse models. Cancer Lett 339: 135-143, 2013.

5. Hruban RH, Adsay NV, Albores-Saavedra J, Anver MR, Biankin AV, Boivin GP, Furth EE, Furukawa T, Klein A, Klimstra DS, et al: Pathology of genetically engineered mouse models of pancreatic exocrine cancer: Consensus report and recommendations. Cancer Res 66: 95-106, 2006.

6. di Magliano MP and Logsdon CD: Roles for KRAS in pancreatic tumor development and progression. Gastroenterology 144: 1220-1229, 2013. 
7. Hingorani SR, Petricoin EF III, Maitra A, Rajapakse V, King C, Jacobetz MA, Ross S, Conrads TP, Veenstra TD, Hitt BA, et al: Preinvasive and invasive ductal pancreatic cancer and its early detection in the mouse. Cancer Cell 4: 437-450, 2003.

8. Wagner EF: Bone development and inflammatory disease is regulated by AP-1 (Fos/Jun). Ann Rheum Dis 69 (Suppl 1): i86-i88, 2010.

9. Ren X, Song W, Liu W, Guan X, Miao F, Miao S and Wang L: Rhomboid domain containing 1 inhibits cell apoptosis by upregulating AP-1 activity and its downstream target Bcl-3. FEBS Lett 587: 1793-1798, 2013

10. Shi R, Peng H, Yuan X, Zhang X, Zhang Y, Fan D, Liu X and Xiong D: Down-regulation of c-fos by shRNA sensitizes adriamycin-resistant MCF-7/ADR cells to chemotherapeutic agents via P-glycoprotein inhibition and apoptosis augmentation. J Cell Biochem 114: 1890-1900, 2013.

11. Jia ZC, Wan YL, Tang JQ, Dai Y, Liu YC, Wang X and Zhu J: Tissue factor/activated factor VIIa induces matrix metalloproteinase-7 expression through activation of c-Fos via ERK1/2 and p38 MAPK signaling pathways in human colon cancer cell. Int J Colorectal Dis 27: 437-445, 2012.

12. Meng Q and Xia Y: c-Jun, at the crossroad of the signaling network. Protein Cell 2: 889-898, 2011.

13. Lappas M, Riley C, Lim R, Barker G, Rice GE, Menon R and Permezel M: MAPK and AP-1 proteins are increased in term pre-labour fetal membranes overlying the cervix: Regulation of enzymes involved in the degradation of fetal membranes. Placenta 32: 1016-1025, 2011.

14. Liu X, Li Q, Dowdell K, Fischer ER and Cohen JI: VaricellaZoster virus ORF12 protein triggers phosphorylation of ERK1/2 and inhibits apoptosis. J Virol 86: 3143-3151, 2012.

15. Eferl R and Wagner EF: AP-1: A double-edged sword in tumorigenesis. Nat Rev Cancer 3: 859-868, 2003.

16. Bailey JM, Hendley AM, Lafaro KJ, Pruski MA, Jones NC, Alsina J, Younes M, Maitra A, McAllister F, IacobuzioDonahue CA, et al: P53 mutations cooperate with oncogenic Kras to promote adenocarcinoma from pancreatic ductal cells Oncogene 441: 1-7, 2015.

17. Han H and Von Hoff DD: SnapShot: Pancreatic cancer. Cancer Cell 23: 424-424.e1, 2013.

18. Stylianopoulos T, Martin JD, Chauhan VP, Jain SR, DiopFrimpong B, Bardeesy N, Smith BL, Ferrone CR, Hornicek FJ, Boucher Y, et al: Causes, consequences, and remedies for growth-induced solid stress in murine and human tumors. Proc Natl Acad Sci USA 109: 15101-15108, 2012.

19. Tabernero J, Chiorean EG, Infante JR, Hingorani SR, Ganju V, Weekes C, Scheithauer W, Ramanathan RK, Goldstein D, Penenberg DN, et al: Prognostic factors of survival in a randomized phase III trial (MPACT) of weekly nab-paclitaxel plus gemcitabine versus gemcitabine alone in patients with metastatic pancreatic cancer. Oncologist 20: 143-150, 2015.

20. Sherman MH, Yu RT, Engle DD, Ding N, Atkins AR, Tiriac H Collisson EA, Connor F, Van Dyke T, Kozlov S, et al: Vitamin $\mathrm{D}$ receptor-mediated stromal reprogramming suppresses pancreatitis and enhances pancreatic cancer therapy. Cell 159: 80-93, 2014.
21. Guerra C, Schuhmacher AJ, Cañamero M, Grippo PJ, Verdaguer L, Pérez-Gallego L, Dubus P, Sandgren EP and Barbacid M: Chronic pancreatitis is essential for induction of pancreatic ductal adenocarcinoma by K-Ras oncogenes in adult mice. Cancer Cell 11: 291-302, 2007.

22. Fukuda A, Wang SC, Morris JP IV, Folias AE, Liou A, Kim GE, Akira S, Boucher KM, Firpo MA, Mulvihill SJ, et al: Stat 3 and MMP7 contribute to pancreatic ductal adenocarcinoma initiation and progression. Cancer Cell 19: 441-455, 2011.

23. Sankaran SJ, Xiao AY, Wu LM, Windsor JA, Forsmark CE and Petrov MS: Frequency of progression from acute to chronic pancreatitis and risk factors: A meta-analysis. Gastroenterology 149: 1490-1500.e1, 2015.

24. Carrière C, Young AL, Gunn JR, Longnecker DS and Korc M: Acute pancreatitis accelerates initiation and progression to pancreatic cancer in mice expressing oncogenic Kras in the nestin cell lineage. PLoS One 6: e27725, 2011.

25. Morris JP IV, Cano DA, Sekine S, Wang SC and Hebrok M: Betacatenin blocks Kras-dependent reprogramming of acini into pancreatic cancer precursor lesions in mice. J Clin Invest 120: 508-520, 2010.

26. Guerra C and Barbacid M: Genetically engineered mouse models of pancreatic adenocarcinoma. Mol Oncol 7: 232-247, 2013.

27. Jafri M, Donnelly B, McNeal M, Ward R and Tiao G: MAPK signaling contributes to rotaviral-induced cholangiocyte injury and viral replication. Surgery 142: 192-201, 2007.

28. Johnson GL and Lapadat R: Mitogen-activated protein kinase pathways mediated by ERK, JNK, and p38 protein kinases. Science 298: 1911-1912, 2002.

29. Garcia MN, Grasso D, Lopez-Millan MB, Hamidi T, Loncle C, Tomasini R, Lomberk G, Porteu F, Urrutia R and Iovanna JL: IER3 supports KRASG12D-dependent pancreatic cancer development by sustaining ERK1/2 phosphorylation. J Clin Invest 124: 4709-4722, 2014

30. Thompson KN, Whipple RA, Yoon JR, Lipsky M, Charpentier MS, Boggs AE, Chakrabarti KR, Bhandary L, Hessler LK, Martin SS, et al: The combinatorial activation of the PI3K and Ras/MAPK pathways is sufficient for aggressive tumor formation, while individual pathway activation supports cell persistence. Oncotarget 6: 35231-35246, 2015.

31. Mace TA, Shakya R, Elnaggar O, Wilson K, Komar HM, Yang J, Pitarresi JR, Young GS, Ostrowski MC, Ludwig T, et al: Single agent BMS-911543 Jak2 inhibitor has distinct inhibitory effects on STAT5 signaling in genetically engineered mice with pancreatic cancer. Oncotarget 6: 44509-44522, 2015.

32. Reichert M and Rustgi AK: Pancreatic ductal cells in development, regeneration, and neoplasia. J Clin Invest 121: 4572-4578, 2011. 